\title{
Students' opinions about the effects of preclinical patient contacts on their learning
}

\author{
Agnes D. Diemers · Diana H. J. M. Dolmans • Maarten G. M. Verwijnen • \\ Erik Heineman · Albert J. J. A. Scherpbier
}

Received: 18 January 2007/ Accepted: 8 May 2007/Published online: 13 July 2007

(C) Springer Science+Business Media B.V. 2007

\begin{abstract}
Several reasons have been given why students should have contacts with real patients early in the undergraduate medical curriculum, i.e., in the preclinical phase. However, it is not clear exactly what effects early patient contacts have with regard to knowledge construction and the development of clinical reasoning skills. We sought students' views of the effects of preclinical real patient contacts on their learning, knowledge construction and development of clinical reasoning. Twenty-four students in the third and last preclinical year of a 6-year undergraduate medical programme were divided into three focus groups which met twice, after which saturation was reached. The discussions were recorded and transcribed. Summaries of the discussions were approved by the students after some modifications. Atlas-ti software was used to create a coding framework resulting in identification of main themes and sub themes. Early patient contacts motivate students to study, help them understand the impact of illness on patients' lives, and enhance professional socialisation and memory processes. Students distinguish between analytic and non-analytic clinical reasoning in connection with real patients. Analytic reasoning involves clinical and basic science knowledge. Non-analytic reasoning involves pattern recognition and is made possible by experiential learning from different patient contacts. The students indicate that seeing real patients early in their training has several positive effects on their learning. The contacts enhance knowledge construction and
\end{abstract}

\footnotetext{
A. D. Diemers $(\bowtie) \cdot$ M. G. M. Verwijnen

Skillslab, Faculty of Health, Medicine and Life Sciences, Maastricht University,

P.O. Box 616, Maastricht 6200 MD, The Netherlands

e-mail: A.Diemers@sk.unimaas.nl
}

D. H. J. M. Dolmans

Department of Educational Development and Research, Maastricht University, Maastricht,

The Netherlands

E. Heineman

Department of Surgery, University Hospital Maastricht, Maastricht, The Netherlands

A. J. J. A. Scherpbier

Institute for Medical Education, Faculty of Health, Medicine and Life Sciences, Maastricht University, Maastricht, The Netherlands 
clinical reasoning. Although our results will have to be validated by quantitative, observational and experimental research, they imply that educational benefits are to be gained from real patient contacts in the preclinical phase of medical education.

Keywords Preclinical patient contacts - Basic science knowledge - Clinical knowledge Experiential knowledge $\cdot$ Clinical reasoning $\cdot$ Analytic reasoning $\cdot$ Non-analytic reasoning

\section{Introduction}

Real patient contacts early in medical education, i.e., in the preclinical phase, have been advocated for several reasons. They are assumed to ease the transition from preclinical to clinical training (Prince et al. 2000; Seabrook 2004), motivate students to learn (Prince et al. 2000), and help them forge their professional identity (O'Brien et al. 2001; Pitkala and Mantyranta 2003). Students have been reported to feel more comfortable performing physical examination (O'Brien et al. 2001) and be more aware of the impact of illness on patients (Cooper et al. 2001; Frank et al. 1996) as a result of seeing real patients. A systematic review of early patient contacts cited positive effects on professional socialisation (Dornan et al. 2006). Reported positive effects also include improved acquisition and retention of knowledge (Prince et al. 2000; Seabrook 2004) and contextualisation of basic science knowledge (O'Brien et al. 2001; Mann 1994) by linking real patients with theory. However, no detailed studies have addressed exactly in what way early patient contacts impact on how students acquire knowledge, use basic science knowledge and integrate basic science and clinical knowledge during clinical reasoning. In a paper in which he synthesised the literature on clinical reasoning Norman (2005) contended that research on clinical reasoning generally focuses on "the processes doctors use to arrive at an initial diagnosis based on history and physical examination', (Norman 2005). He distinguishes basic science knowledge, i.e., formal knowledge, including illness scripts and schemas, and experiential knowledge, i.e., informal knowledge, consisting of a store of exemplars resulting from past experience. Norman suggests that experts resort to basic science knowledge mainly when solving "rare and complex" patient problems but rely mainly on similarity-based reasoning when it comes to common and less complex problems (Norman 2005). Accordingly, Eva made the distinction between analytic and non-analytic clinical reasoning (Eva 2005). Experts use analytic reasoning to explain and understand the mechanisms involved in relating symptoms and diagnosis. Non-analytic reasoning refers to pattern recognition involving comparison of new and past cases, combined with assessing the probability of a new case falling within a particular diagnostic category. Given the strong role of experience in both Eva's and Norman's views on clinical reasoning, patient contacts may have a prominent part to play in expertise development in this area.

Earlier studies of students' patient contacts were mostly limited to the clinical phase of undergraduate education, investigating the effect of student contacts with patients during the clinical phase (Seabrook 2004; Prince et al. 2000); or the assumed effects of preclinical patient contacts (Prince et al. 2000). Manchester implemented PBL in the clinical years of the medical curriculum using paper cases as the starting point for learning, with students seeking clinical experiences linked to the paper cases. Students stated that their clinical experiences helped them elaborate on their knowledge (O'Neill et al. 2002). However, none of the described studies used real patients as the starting point for learning in the preclinical phase of medical education, which may lead to additional effects on student 
learning and more specific on clinical reasoning. Recently, Maastricht Medical School introduced real patient encounters in Year 3 of their preclinical medical PBL curriculum. A first evaluation of the preclinical real patient contacts indicated that according to the students the encounters enhanced integration of theory and practice (Diemers et al. 2007). The limitations of that study were, however, that it elicited only superficial information about the effects of the implementation of preclinical patient contacts on students' learning. Because of the assumed effects of experience with patient contacts on the learning of students and more specifically on the development of their clinical reasoning ability, we conducted a study aimed at in-depth investigation of the effects of preclinical real patient contacts. We sought students' perceptions of the effects of preclinical real patient contacts on their: (1) learning; (2) knowledge construction; and (3) development of clinical reasoning.

\section{Methods}

\section{Setting}

We explored the opinions of students in Year 3 of the 6-year problem-based learning (PBL) curriculum of Maastricht Medical School. Year 3 is the last preclinical year, after which clinical clerkships start in Year 4. In Years 1-2 small group tutorials, using paper patients, are the predominant educational format. A recent curriculum change discarded the paper patient based tutorials in Year 3 and replaced them by real patient encounters in the teaching outpatient clinic in the academic hospital. These patient encounters are used as triggers for learning in the tutorials related to the encounters. Clinical skills are learned during a 5 year clinical skills programme starting in Year 1 and continuing through Year 5.

In Year 3 the students rotate through four 10-week clusters, each dedicated to a different domain of chronic disease (Fig. 1). For logistical reasons all students in Year 3 are divided into four groups which complete the four clusters in different order. The basic educational unit is the tutorial group of 10 students and a tutor, the so-called coach. Coaches are staff members of the disciplines participating in the thematic clusters. Group composition and coaches change every cluster. The weekly recurring PBL cycle (Fig. 2) in Year 3 starts with a session in which students prepare for their upcoming patient encounter by discussing vignettes of the (type of) patient problem they will see in the hospital. During the patient encounter phase, 1-2 days after the tutorial, student pairs see patients with chronic disorders in the teaching outpatient clinic of the University Hospital of Maastricht. The students perform the first part of the consultation, i.e. they take a history and examine the patient. After that they consult the patient's attending physician, the so-called clinical supervisor, and together they return to the patient to complete the consultation. Subsequently, the clinical supervisor discusses the encounter with the students, helps them derive learning objectives and gives feedback on their performance. The students pursue

\begin{tabular}{|l|c|c|c|c|}
\hline \multicolumn{5}{|c|}{ Year 3: Chronic Disease } \\
\cline { 1 - 4 } & Weeks 1-10 & Weeks 11-20 & Weeks 21-30 & Weeks 31-40 \\
\cline { 1 - 4 } Student groups & Abdomen & Locomotor & Circulation and lungs & Psychomedical \\
\cline { 1 - 4 } Group 1 & Locomotor & Circulation and lungs & Psychomedical & Abdomen \\
\hline Group 2 & Circulation and lungs & Psychomedical & Abdomen & Locomotor \\
\hline Group 3 & Psychomedical & Abdomen & Locomotor & Circulation and lungs \\
\hline Group 4 & \multicolumn{4}{|l}{}
\end{tabular}

Fig. 1 Logistics of Year 3: four groups of students attend four subject clusters in different order. At the end of the year each group has completed the same programme 


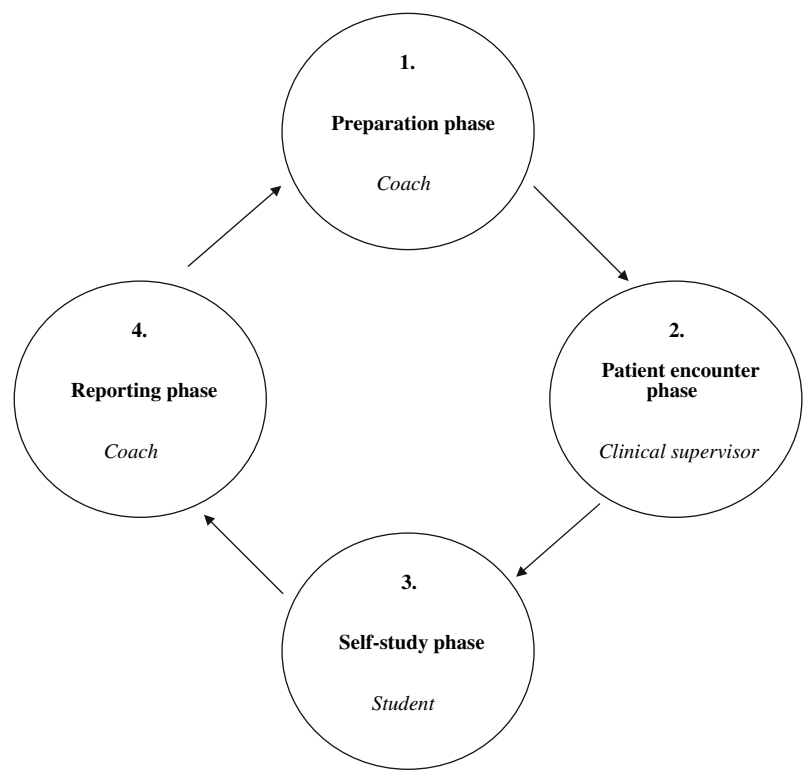

Fig. 2 The four phases of the PBL-cycle

the learning objectives through self study and present and discuss their findings in the next tutorial, taking place 1 week after the first (preparatory) session (Fig. 2).

Research methodology

In exploring students' perceptions of the effects of early patient encounters, we focused on students perceptions of how their learning was affected by the contacts and why they thought this was so. Students' opinions were explored in focus group interviews. This method has been shown to be effective in eliciting a rich variety of opinions from groups (Kitzinger 1995). Participants in focus groups share, compare, and explore their ideas and experiences about specific issues and by doing so generate their own interpretations (Morgan 1998). They are asked to contemplate their own ideas in the context of the ideas of others (Patton 1987).

\section{Subjects}

All students in Year 3 in the academic year 2004-2005 were invited to participate in a focus group. An email was sent to all the students, explaining the purpose and procedure of the study and inviting volunteers to participate. Next, all the students were approached in person by the principal researcher during skills training sessions at the end of the academic year 2004-2005, when the students had completed almost all of the four clusters in Year 3. Three focus groups, each consisting of eight students, were composed by purposive sampling. Purposive sampling was used to achieve a relevant mix of educational experiences. It involved selecting the students in chronological order of response. The selection criteria required that each focus group should consist of four pairs of students who were attending different clusters at the time of the interview and who had preferably not been in 
the same tutorial group at any time during the year. In this way we aimed to achieve representation of experiences with as many different tutorial groups and coaches as possible to maximise diversity of experiences in the groups and thereby stimulate discussion (Barbour 2005). This resulted in two focus groups of 6 female students (75\%) and 2 male students $(25 \%)$ and one focus group of 5 female students $(62.5 \%)$ and 3 male students (37.5\%). Gender distribution in the cohort was $201(70 \%)$ female and 90 (30\%) male students. Participation was voluntary and students received a small compensation. Students were assured that the results would be reported anonymously. Currently, educational studies reporting students' opinions do not require approval from the ethics committee in the Netherlands.

\section{Instruments}

The focus groups were asked to answer open-ended questions about three topics (Appendix A):

1. The effects of real patient contacts on their learning;

2. The effects of real patient contacts on their knowledge construction;

3. The effects of real patient contacts on their clinical reasoning.

\section{Procedure}

Each focus group met twice for $90 \mathrm{~min}$. A third meeting was not considered necessary since by the end of the second meeting no new points of view were emerging, i.e., saturation had been reached. A second focus group meeting was considered necessary, because in the debriefing sessions and in the initial analysis of the summaries of the first focus group meetings, topics were identified that were clearly in need of further clarification or more in depth discussion. The first moderator (MV) of the focus group sessions had more than 30 years of experience with small group education. He guided the interview and stimulated the discussion by asking additional questions and encouraging all participants to contribute to the discussion. The assistant moderator (AD), the principal researcher, observed, took notes and audio taped the interviews. At the beginning of each interview MV stated the rules of the meeting: participants were invited to share and compare their views and ideas, and modify them in light of the ideas of other participants. They were informed that analysis and reporting of the results would be anonymous. In debriefing sessions following each interview, the moderators shared their impressions of the session. They discussed whether appropriate information had been gathered and whether it was necessary to adjust the questions for the next interview in light of the research questions. Initial identification of major themes was established during these sessions.

The recordings of all the interviews were transcribed literally by the principal researcher. Summaries were written and submitted to the participants for approval. The students were asked to send comments and suggestions for modifications to the moderators before the next meeting. All the students responded and approved the summaries after some minor adjustments had been made.

\section{Analysis}

The transcripts of the interviews were imported into the software program ATLAS-ti (Muhr 1997). Initial coding of text fragments based on content was done through multiple coding 
by two researchers (AD and AS) independently (Barbour 2001). The coding framework was discussed by the researchers and modified until agreement was reached. The transcripts were re-read and modifications in the coding were established in accordance with the agreed coding framework. In the same way agreement was reached on a thematic framework, consisting of main themes and sub themes (Table 1). The main themes correspond with the main research questions discussed by the focus groups, with new themes that were raised by the participants during the discussions and with analytical themes that emerged during the analysis (Ritchie and Spencer 1994). The sub themes resulted from our analysis of the data using the coding framework. In this way we identified new themes and sub themes during analysis of the data alongside the main themes established through our research questions (Melia 1997). All text units belonging to a code of the coding framework were sorted according to the themes of the thematic framework. Text units were re-read to be sure that no "emerging themes" had been overlooked. Illustrative quotes were selected, translated, and, when necessary, edited to improve clarity without changing meaning.

\section{Results}

We present the results for the main themes and the sub themes (Table 1) and illustrate them with quotations from the focus group sessions.

The effects of real patient contacts on student learning

\section{Motivation to study}

Taking part in a real consultation with a real patient is a strong incentive for students to prepare for the contact. They are keen to avoid failure in front of a patient and take the responsibility for their part in the process of patient care very seriously. No paper patient can provoke comparable, powerful feelings.

"When you know you are going to see a real patient, you study really hard, because you want to do your best and not be stuck for answers. . . . you don't have the same sense of urgency with paper patients.', (S7.2.1)

Table 1 Main themes and subthemes discussed during the focus group interviews

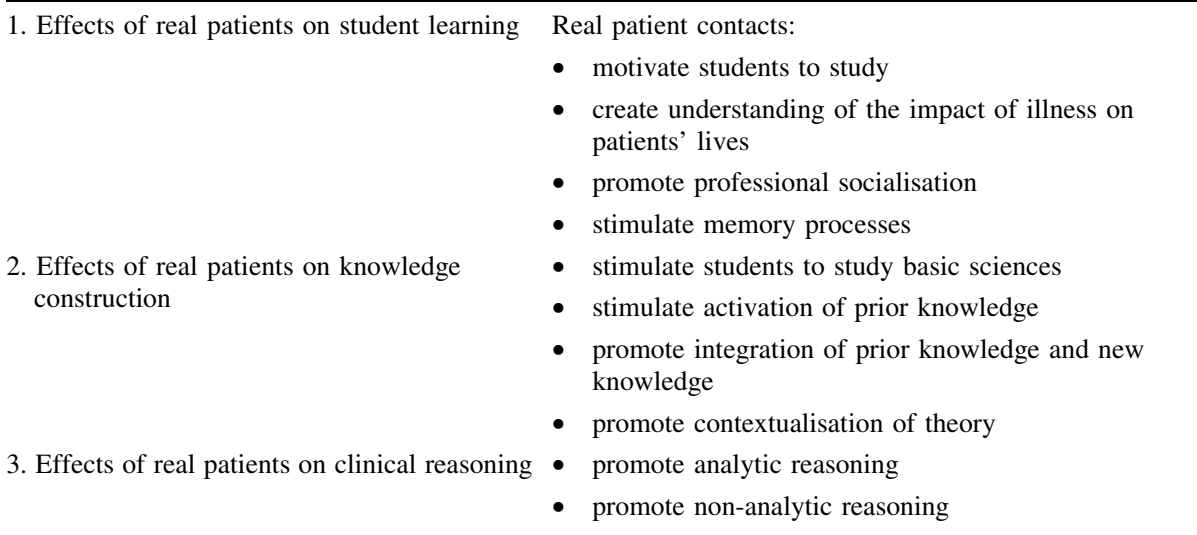


Before you go to the outpatient clinic, you feel stimulated to study because you are given responsibility." (S4.3.1) "It is not as if you have to make real decisions but it is your responsibility to conduct the consultation and treat the patient with respect and you can actually mean something to that patient. (S2.3.1)

Seeing real patients excites students' curiosity. It is a more powerful motivator to study the literature than paper patients could ever be.

When I have seen a real patient I am much more motivated to study than after discussing a paper patient. (S5.1.1)

\section{Understanding the impact of illness}

Students say that the contacts are a real eye opener with respect to the impact of illness on patients' lives. They see that the limitations experienced by patients due to symptoms can have a farther reaching impact on patients' lives than they had thought possible.

I learn a lot about what a disease does to a patient's life. You see that even the most insignificant, trivial disorders can have a huge impact. You don't realise that when you only read about it. For instance when you read in textbooks about Dupuytren's contracture. And then, when you see it for real, you think: yes, I would be really fed up if I had that. (S2.2.1)

Because the students see many different patients, they come to realise that illness affects different patients in different ways. This can help them put patients' stories into perspective.

I once saw a lady in the outpatient clinic who . . . thought her disorder was severely limiting, whereas earlier I had seen a man with heart failure who had only a few more weeks left to live. That makes you put things somewhat into perspective for this lady. (S1.2.2)

\section{Professional socialisation}

Students report that patient encounters help them get used to dealing with real patients and their future professional role as a doctor. In their opinion, this cannot be achieved by paper patients.

When I saw my first patient in the outpatient clinic, I thought like "strange a real patient', But after a while it makes no difference. But that takes time. And getting used to the role of doctor-patient. You don't learn that from paper cases. (S1.2.1)

\section{Memory processes}

Students say that they remember more about a disease when they see a real patient than when they only read about it. Seeing real patients intensifies self study and efforts to link theory and patients. This promotes retention and facilitates retrieval of knowledge.

Students' active involvement in consultations with real patients when they take a history and examine the patient encourages them to think actively about patients' problems, which in turn may enhance storage and retrieval of knowledge. 
When you have conducted the consultation yourself, you remember the patient and the things you or the supervisor have said about it much better. (S7.1.2)

When the coach asks critical questions you are stimulated to think about where your knowledge is stored or something. You know which drawer to open. And that makes it easier to remember things. (S7.3.2)

The students say that memorable cases, i.e., patients who arouse strong emotions or patients who make a specific impression, are easier to remember.

Some patients you will never forget." (S3.3.2) "They have to be really different, trigger an emotional response, no matter what sort, but the impression has to be powerful. (S7.3.2)

The power to bolster knowledge storage and retrieval is an important distinction between real patients and paper patients. Students do not remember much about paper patients because they make less of an impression.

Paper patients make less of an impression on you. (S8.2.1)

The same applies for simulated patients. Students are aware of participating in role play in consultations with simulated patients. The patients' emotions do not seem real to them. Another drawback of simulated patients is that some physical signs are difficult to simulate realistically, which reinforces students' sense of play-acting.

Somehow you just don't believe in simulated patients. You know it is just play acting. You do try and get into the part and all that, but it is different all the same." (S4.2.1) "In a simulated patient you cannot see the impression. You hear everything. But when his ankle is broken, the ankle is not red or swollen. All he can simulate is that he is unable to stand on it. It is just not complete. A real patient has everything, or in any case has real complaints and that like creates the picture. With a simulated patient there will always be a gap. (S7.3.2)

The effects of real patient contacts on knowledge construction

\section{Basic sciences}

Students perceive basic science knowledge as a prerequisite for understanding how patient problems are related to underlying concepts. Seeing real patients motivates students to brush up on their basic science knowledge.

With real patients you are sort of forced to use your old knowledge of physiology, anatomy. (S2.2.2)

It is like this, physiology, when you understand it, it becomes much easier to learn about pathology. Then you have some idea of where the patient's symptoms are coming from. (S4.2.2)

\section{Prior knowledge}

Real patients stimulate not only acquisition of new knowledge but also activation of existing knowledge. Forging new links between new and prior knowledge strengthens students' knowledge networks. 
Because a patient makes you want to study new things, you are more motivated. So then you activate the knowledge you already have and the new information you are going to learn. (S3.2.2)

You have to know both. New knowledge and old knowledge. When they are both separate, they are of no use to you." (S5.3.2) "You find that you can place things. That you don't think it strange that a patient should have a certain complaint because you can relate it to your basic science knowledge from year 1 and year 2. (S2.3.2)

\section{Integration of prior knowledge with new knowledge}

Integrating prior and new knowledge increases students' awareness that they are connecting pieces of information and creating a coherent knowledge network.

You know a certain disease because you have learned the theory. And then you see a real patient who fits into that and then, well you get the full picture. (S2.3.2)

You are better able to see the whole picture. As if some things just fall into place, whereas first they just floated around loosely inside my head. (S3.2.2)

Students feel that this process happens unconsciously and automatically. Critical questions asked by the coach or other students make them aware of this process and this reinforces integration of new and old knowledge.

You are like constructing new knowledge on top of your old knowledge, making links. In my opinion it is a passive process. (S2.2.2)

Yes it is an automatic thing. And that you start to think much more for yourself when you are studying. (S8.2.2)

When I am studying I am not really aware of it. Then I think that you are not consciously making links, like for yourself, and when you have to explain things to some-one else or you have to answer a question, that is the time when you are actively engaged in it. And in those cases, at least for me, it is rather more productive. (S7.2.2)

\section{Contextualisation of theory}

Experiences with real patients improve students' comprehension of subject matter, because real patients are powerful illustrations of pathological mechanisms described in textbooks.

... when you have studied a certain disease, pathology, and you have seen a patient with the disease, then you can make the link. ... When I read about it in a book, I can suddenly understand the patient. (S4.2.2)

For instance, you read in textbooks that some-one has difficulty breathing when they lie down flat. But, well you don't really see it happening. And when you have seen a patient in the outpatient clinic who lies down on the examination table and turns all red, then you see it immediately before you when you read about it afterwards. (S1.2.1)

Students say that real patient problems help them form a more complete picture of disease and underlying mechanisms. They also obtain a more holistic view of patients, which goes beyond the organ system(s) involved in the presenting problem.

... when you see real patients more and more pieces of the jigsaw start to fall into place. Because with paper cases, then you, like we are doing the lungs, you just focus on the lungs. And now, in practice, I find that it is not just about the lungs, it is about 
the whole system and everything else that is involved. Much more complete when you see real patients. (S8.2.1)

Due to contacts with different patients with similar disorders students discover that diseases often do not conform to the typical picture or course described in textbooks but have different dimensions. This furthers comprehension as well.

Another thing that makes a patient easy to remember is when something does not fit with what is in the book. And again that is actually really helpful to better understand both the real patient and pathophysiology. (S8.1.2)

When you see a real patient, you see that things are not always exactly according to the description of the disease. It can look very differently. (S5.2.2)

And you start to see that a disease can have different manifestations. One patient has different symptoms from another one. And when you read it (disease) in a book, you think, yes ... But when you are looking at a real patient then for instance he does not have the first three symptoms but he does have the last four ones. That is also possible. And then you realise that a disease has many dimensions because you see many different patients (S1.3.2)

Students say that simulated patients always portray a typical patient, i.e., the standard textbook presentation of a particular disease. That is why simulated patients add nothing to what they learn from real patients. The same comment is made about paper patients.

Simulated patients always have symptoms exactly by the book (S3.1.2)

Most paper patients are very typical, very characteristic. (S5.2.2)

With a real patient you have to find things out for yourself and ask questions. With paper patients you don't even have to find things out, for it is all written down in the paper case, all the information you need. (S2.1.2)

The effects of real patient contacts on clinical reasoning

With regard to clinical reasoning skills, students say that initially they ask questions from memorised checklists. After a while they are able to abandon these lists and move on to more deliberately structured clinical reasoning. The fact that both their basic science knowledge and their clinical knowledge increase during the year facilitates this transition.

For example in year 1, when I had to learn about a disease I would memorise lists of symptoms and now I try to find out what is going on and what causes the symptoms. And then I can explain them, and then I no longer need that list. I have more background knowledge now. Then I only learned lists by heart, and I have totally stopped doing that now. (S4.2.2)

... I could really think in certain steps. It was a chronic patient with multiple symptoms and then you find that you can start to sort of order them. Well, I know for sure that at the beginning of third year I would definitely not have been able to do that. (S2.2.2)

During the year students become increasingly able to integrate the knowledge from the different clusters. One student described how he used knowledge about circulation and lungs to understand symptoms of impotence in a patient he saw in the cluster on abdominal region.

For instance I saw a man with complaints about impotence and he proved to have a history of cardiac complaints and vascular problems. And then you think like, well, but that can also occur there so that you get those problems. And in that way, eh, you 
use different things, like in the urology outpatient clinic you saw this and in cardiology you learned that, well, but those things can also be combined. (S4.3.2)

\section{Analytic reasoning}

The analysis of the discussions revealed that students use two types of clinical reasoning. The first one involves explaining and understanding relations between signs and symptoms and disease. To this end students use what they know about pathological mechanisms of disease and basic science. This is a conscious process, which is frequently triggered by questions from teachers or peers.

When for instance you have something pathological and you say this is the physiology we have just discussed that or it is assumed that it is known, then you say, eh, well the patient is vomiting, what might cause this? Yes, you know physiology, you know how food is swallowed normally, how it is digested and everything. Why should a vomiting reflex occur? Suppose large chunks of food come out. How is that possible? Which pathological mechanism would be more likely than another one? You start to reason from physiology to find out about pathology. (S4.3.2)

\section{Non-analytic reasoning}

The second method of clinical reasoning involves recognising patterns in patient problems. This is often an unconscious process which starts as soon as students have seen one patient with a certain problem.

Suppose you see another patient with a complaint that is sort of similar, then that rings a bell like hey this looks familiar. It might be this and that, this I have seen before. I think you are not conscious of it. (S6.2.2)

A little boy had pneumonia and I had done that together with the GP. And the next time I was at the GPs, it was winter again, so another boy turns up with very similar symptoms. And then I immediately thought well, that might be pneumonia as well. And it actually turned out that it was and I had really spotted it. (S5.3.2)

\section{Conclusions and discussion}

The results of our study indicate that in the students' opinion their learning benefits from real patient contacts. The results furthermore demonstrate that, according to the students, different kinds of knowledge constructions are emerging and different ways of clinical reasoning, i.e., problem solving strategies, are developing.

The results of our study are consistent with earlier findings about the effect of real patients on student learning: the real patient leads to better acquisition and retention of knowledge (Prince et al. 2000; Seabrook 2004), motivates learning (Prince et al. 2000), vividly demonstrates the impact of disease on patients' lives (Cooper et al. 2001; Frank et al. 1996), and stimulates the process of professional socialisation (Dornan and Bundy 2004; Pitkala and Mantyranta 2003). However, our study revealed additional effects of preclinical patient contacts on student learning. According to the students analytic as well as non-analytic reasoning strategies were being developed. Experts have been shown to use both types of reasoning, either separately or in combination, when addressing clinical problems (Eva 2005; 
Norman and Brooks 1997; Elstein and Schwarz 2002; Bowen 2006). Novices on the other hand have been found to use analytic reasoning strategies in favour of non-analytic strategies because they do not have enough experiential knowledge (Bowen 2006). Conversely, recent studies showed that pattern recognition is a powerful tool in diagnostic accuracy in experts as well as in novices after they had been explicitly instructed to use pattern recognition, especially when used in combination with other reasoning strategies (Coderre et al. 2003; Ark et al. 2007). The results of our study suggest that medical students, who are clearly novices, are able to develop and use both ways of clinical reasoning even without explicit instruction but with real patients as the driving force for their learning. With regard to analytic reasoning, students argue that real patient encounters stimulate them to study basic science. Combined use of basic science and clinical knowledge helps them to explain connections between signs, symptoms and diagnosis. When students use analytic reasoning, they give meaning to the symptoms of the patient but also to their basic science knowledge. This is supposed to enhance recall and retention (Woods et al. 2005). With regard to nonanalytic reasoning, it seems that students think that practice with multiple patient examples helps them build experiential knowledge structures. Experiential knowledge networks are built upon examples derived from past experience. Both kinds of clinical reasoning, analytic and non-analytic, are needed to ensure transfer of knowledge and problem solving strategies so that they can be used to resolve new patient problems (Eva 2005; Woods et al. 2005).

Students indicate that coaches and clinical supervisors have an important role to play in relation to the development of their clinical reasoning skills. Students said that they started to actively use clinical reasoning and thinking as a result of their teachers or peers asking critical questions. This supports findings reported in the literature that students did not automatically of their own accord study the pathophysiology underlying patients' problems or make links across patient problems (van de Wiel et al. 1999; Eva 2005).

In conclusion, the results of this study appear to demonstrate that students indicate that they develop both analytic and non-analytic types of clinical reasoning as a result of real patient contacts early in medical education, i.e., in the preclinical phase. According to the students, paper patients and simulated patients have less learning potential for the development of clinical reasoning in this phase of their medical education. For medical education the implication appears to be that students should be given ample opportunities not only to acquire formal knowledge to develop analytic reasoning skills but also to build experience to collect many exemplars of patient cases, i.e., to amass informal knowledge, for use in non-analytic reasoning (Norman 2006).

This study has some limitations. First of all we investigated the effects of preclinical patient contacts by enquiring into students perceptions of this educational format. This means that it would be desirable to complement this qualitative research with quantitative research to determine the generalisability of our findings. Even though our results are partly consistent with earlier findings, additional research should validate students' opinions about the processes of analytic and non-analytic reasoning. Secondly, such quantitative studies should be performed among randomly selected larger groups of students. Because the participants in this study were not selected at random, it cannot be excluded that the study sample consisted of mainly highly motivated students who volunteered. This may have influenced our results. Although we used focus groups to explore students' opinions, we did not analyse the group processes during these group sessions. The reason we used focus groups rather than one-to-one interviews was to create the possibility for the participants to share ideas, views and meanings and change them or develop new ones if they thought this was appropriate. This is not possible in individual interviews and adds to the richness of the data. We did not examine agreements or disagreements between group 
members or the influence of group processes on the discussion. This may have caused us to overlook unspoken views or ideas that were not shared because of certain group dynamics during the interviews.

Finally, some students knew the main moderator as the head of the Skillslab. Although this may have led to students withholding views or ideas, we feel such an effect is likely to be minimal since the research topic was not directly related to the Skillslab programme. Also, the analysis of the data revealed no indications of such an effect.

Several recommendations for further research may be derived from the results. The teachers' contribution to early patient contacts deserves further investigation, the more so since students consider the teacher's role to be crucial, especially in activating clinical reasoning processes. Finally, future research should focus on validation of the results of this qualitative study by quantitative as well as observational and experimental research.

Acknowledgements The authors thank the students of Maastricht Medical School for their participation in this study. They also thank Mereke Gorsira for editing the final version. Ethical approval: Ethical approval was not required for this study.

\section{Appendix A}

Interview questions

1. What are the effects of real patients vs. paper patients and simulated patients on your learning?

- in relation to knowledge acquisition

- in relation to use of knowledge

2. What happens in Year 3 with the knowledge you have at the start of the year?

- How do you use it?

- How is that stimulated?

- When do you use it?

- Why do you use it?

- How do you know that you are using it, how can you tell?

- How can you tell that your basic science knowledge is deficient (as was mentioned in the first interview round)?

- How can you tell that your basic science knowledge is barely adequate?

3. How is your new knowledge integrated with your existing knowledge?

- Does integration occur at all?

- How can you tell?

Where does this occur?

- What do you do with that when it happens?

- How do paper patients compare to real patients in regard to this integration?

4. a. How do you make use of your patient encounters?

- When you are studying

- During other clinical encounters

- In other educational settings

b. The same question is asked with regard to:

- other students' patient encounters (presentations)

- examples given by the coach or clinical supervisor 


\section{References}

Ark, T. K., Brooks, L. R., \& Eva, K. W. (2007). The benefits of flexibility: The pedagogical value of instructions to adopt multifaceted diagnostic reasoning strategies. Medical Education, 41, 281-287.

Barbour, R. S. (2001). Checklists for improving rigour in qualitative research: A case of the tail wagging the dog. British Medical Journal, 322, 1115-1117.

Barbour, R. S. (2005). Making sense of focus groups. Medical Education, 39, 742-750.

Bowen, J. L. (2006). Educational strategies to promote clinical diagnostic reasoning. The New England Journal of Medicine, 355(21), 2217-2225.

Coderre, S., Mandin, H., Harasym, P. H., \& Fick, G. H. (2003). Diagnostic reasoning strategies and diagnostic success. Medical Education, 37, 695-703.

Cooper, H. C., Gibbs, T. J., \& Brown, L. (2001). Community-orientated medical education: Extending the boundaries. Medical Teacher, 23(3), 295-299.

Diemers, A. D., Dolmans, D. H. J. M., Van Santen, M., Van Luijk, S. J., Janssen-Noordman, A. M. B., \& Scherpbier, A. J. J. A. (2007). Students' perceptions of early patient encounters in a PBL curriculum: a first evaluation of the Maastricht experience. Medical Teacher, 29 (accepted for publication).

Dornan, T., \& Bundy, C. (2004). What can experience add to early medical education? Consensus survey. British Medical Journal, 329, 834-837.

Dornan, T., Littlewood, S., Margolis, S. A., Scherpbier, A., Spencer, J., \& Ypinazar, Y. (2006). How can experience in clinical and community settings contribute to early medical education? A BEME systematic review. Medical Teacher, 28(1), 3-18.

Elstein, A. S., \& Schwarz, A. (2002). Clinical problem solving and diagnostic decision making: Selective review of the cognitive literature. British Medical Journal, 324, 729-732.

Eva, K. W. (2005). What every teacher needs to know about clinical reasoning. Medical Education, 39, 98-106.

Frank, D., Handfield-Jones, R., Dawson, D. J., Russel, R., Steinert, Y., Boillat, M., Yaffe, M., Guzder, J., \& Keyserlingk, E. (1996). An integrated curriculum for teaching preparatory clinical skills at a traditional medical school. Teaching and Learning in Medicine, 8(1), 4-9.

Kitzinger, J. (1995). Introducing focus groups. British Medical Journal, 311, 299-302.

Mann, M. P. (1994). A light at the end of the tunnel: The impact of early clinical experiences on medical students. New Orleans, LA: Annual Meeting of the American Educational Research Association.

Melia, K. M. (1997). Producing "plausible stories": Interviewing student nurses. In G. Miller \& R. Dingwall (Eds.), Context and method in qualitative research (pp. 26-36). London: Sage.

Morgan, D. L. (1998). The focus group guidebook. London: Sage Publications.

Muhr, T. (1997). ATLAS/ti-Visual qualitative data analysis-management-model building in education, research and business. Release 4.1 ed. Berlin: Scientific Software Development.

Norman, G. R., \& Brooks, L. R. (1997). The non-analytical basis of clinical reasoning. Advances in Health Sciences Education, 2, 173-184.

Norman, G. (2005). Research in clinical reasoning: Past history and current trends. Medical Education, 39 , 418-427.

Norman, G. (2006). Building on experience-the development of clinical reasoning. New England Journal of Medicine, 355(21), 2251-2252.

O’Brien-Gonzales, A., Blavo, C., Barley, G., Steinkohl, D. C., \& Loeser, H. (2001). What did we learn about early clinical experience? Academic Medicine, 76(4, supplement), S49-S54.

O'Neill, P. A., Willis, S. C., \& Jones, A. (2002). A model how students link problem-based learning with clinical experience through "elaboration'. Academic Medicine, 77(6), 552-561.

Patton, M. Q. (1987). How to use qualitative methods in evaluation. Newbury Park, CA: Sage Publications.

Pitkala, K. H., \& Mantyranta, T. (2003). Professional socialization revised: medical students' own conceptions related to adoption of the future physician's role-a qualitative study. Medical Teacher, 25(2), $155-160$.

Prince, K. J. A. H., Van de Wiel, M., Scherpbier, A. J. J. A., Van der Vleuten, C. P. M., \& Boshuizen, H. P. A. (2000). Qualitative analysis of the transition from theory to practice in undergraduate training in a PBL-medical school. Advances in Health Sciences Education, 5(2), 105-116.

Ritchie, J., \& Spencer, L. (1994). Qualitative data analysis for applied policy research. In A. Bryman (Ed), Analyzing qualitative data (pp. 173-194). London: Routledge.

Seabrook, M. (2004). Clinical students' initial reports of the educational climate in a single medical school. Medical Education, 38(6), 659-669. 
Van de Wiel, M. W. J., Schaper, N. C., Scherpbier, A. J. J. A., van der Vleuten, C. P. M., \& Boshuizen, H. P. A. (1999). Students' experiences with real-patient tutorials in a problem-based curriculum. Teaching and Learning in Medicine, 11(1), 12-20.

Woods, N. N., Brooks, L. R., \& Norman, G. R. (2005). The value of basic science in clinical diagnosis: creating coherence among signs and symptoms. Medical Education, 39(1), 107-112. 